\title{
An ACA-MFS approach for the analysis of sound propagation in sonic crystals
}

\author{
L. Godinho ${ }^{1,2}$, D. Soares Jr. ${ }^{3}$ \& P. G. Santos ${ }^{1}$ \\ ${ }^{1}$ Department of Civil Engineering, University of Coimbra, Portugal \\ ${ }^{2}$ ISISE, University of Coimbra, Portugal \\ ${ }^{3}$ Department of Structural Engineering, \\ Federal University of Juiz de Fora, Brazil
}

\begin{abstract}
Some efficient methods are available in the literature to solve the problem of sound propagation in the presence of sonic crystal structures, such as those based in the Multiple Scattering Theory (MST) or in the Finite Element Method (FEM). More recently, the Method of Fundamental Solutions (MFS) and the Boundary Element Method (BEM) have also been applied for that purpose. In this paper, a new strategy based on the use of the MFS is proposed to tackle problems of sound propagation around 3D sonic crystals with constant geometry along the axis of the scatterers $(2.5 \mathrm{D})$. The problem is solved in the frequency domain, and the sound field is synthesized as a summation of simpler $2 \mathrm{D}$ problems. To allow the solution of large-scale problems, with a high number of scatterers, an Adaptive-CrossApproximation approach is proposed and incorporated in the MFS algorithm, rendering the calculation much faster and with very significant savings in terms of computational requirements. Examples are presented, and the calculation times and RAM requirements are compared with those provided by a classic MFS formulation.

Keywords: sonic crystal, frequency domain, adaptive-cross-approximation, $2.5 \mathrm{D}$ problems.
\end{abstract}

\section{Introduction}

Different aspects of the behavior of sonic crystals have been studied in published works, some of which are essentially theoretical, while others focus some potential practical applications. In the first group, aspects such as the influence of the so 
called point defects (Wu et al. [1]) or the existence of waveguides in which the sound propagates with low attenuation (Vasseur et al. [2]) can be mentioned. In the field of the practical uses of sonic crystals, one which may be regarded perhaps as the most promising is precisely their use for the selective attenuation of sounds, for example as traffic noise barriers (Sánchez-Pérez et al. [3]). A recent work on this topic (Castiñeira-Ibáñez et al. [4]) has addressed the classification of sonic crystal barriers in terms of the relevant European standards for the determination of the intrinsic characteristics of acoustic barriers.

Regarding numerical modeling of these structures, some studies can also be found in the literature. In [5], a multiple-scattering approach is used to analyze the acoustic wave propagation in periodic composites consisting of solid spherical scatterers in a fluid host. Also [6] proposed an extension of the multiple-scattering theory for elastic waves. Application of the Plane Wave Expansion method to study the elastic wave band gaps of steel inclusions embedded in a plastic matrix is found in [7]. The application of the MFS to analyze a sonic crystal composed of circular cylinders was proposed by Martins et al. [8]. In that work, the authors addressed the application for traffic noise attenuation in a 2D approach, with clear advantages in problem discretization and computational cost when compared to the BEM and FEM approaches. In the formulation, the scatterers were considered as rigid elements. Later, Santos et al. [9] extended this formulation to allow considering elastic shell scatterers.

The present paper aims to test the application of an MFS formulation for the $3 \mathrm{D}$ sound propagation analysis through sonic crystals structures. Due to the usual geometry of sonic crystals, which exhibit a constant cross-section along one direction, a 2.5D model is here proposed for that purpose. To allow the analysis of very large structures, with several hundreds or thousands of scatterers, an Adaptive Cross Approximation (ACA) (Rjasanow [10]) strategy together with hierarchical matrices (Bebendorf [11]) is linked to the MFS, allowing a very efficient model to be setup.

The paper is organized as follows: first the theoretical formulation will be presented in terms of the governing equations and of the 2.5D formulation; then, the MFS formulation is briefly described; the main aspects of the application of the ACA and hierarchical matrices are then highlighted; finally, a number of examples is presented illustrating the efficiency and correctness of the predictions computed using the ACA-MFS algorithm proposed here.

\section{Mathematical formulation}

\subsection{Governing equation}

It is usual to consider that the propagation of sound in a fluid space, in the frequency domain, can be mathematically represented by the Helmholtz equation. This equation has the usual form

$$
\nabla^{2} P+k^{2} P=0
$$


where $\nabla^{2}=\frac{\partial^{2}}{\partial x^{2}}+\frac{\partial^{2}}{\partial y^{2}}+\frac{\partial^{2}}{\partial z^{2}}$ for 3D and $\nabla^{2}=\frac{\partial^{2}}{\partial x^{2}}+\frac{\partial^{2}}{\partial y^{2}}$ for 2D problems, $P$ is the acoustic pressure, $k=\omega / c, \omega=2 \pi f, f$ is the frequency, and $c$ is the propagation velocity within the acoustic medium.

Considering the 3D Helmholtz equation, assuming a constant geometry along the $\mathrm{z}$ axis, and applying a spatial Fourier transform along that axis, the $2.5 \mathrm{D}$ Helmholtz equation can be obtained as a function of the axial wavenumber $\left(k_{z}\right)$ as

$$
\left(\frac{\partial^{2}}{\partial x^{2}}+\frac{\partial^{2}}{\partial y^{2}}\right) p\left(k_{z}\right)+\left(k^{2}-k_{z}^{2}\right) p\left(k_{z}\right)=0,
$$

where $p\left(k_{z}\right)$ is the spatially transformed pressure field; $k_{f}{ }^{2}=k^{2}-k_{z}^{2}$ can be seen as the effective wavenumber. The complete $3 \mathrm{D}$ field can be recovered by considering the inverse Fourier transform, so that the $3 \mathrm{D}$ pressure can be calculated as

$$
P=\int_{-\infty}^{+\infty} p\left(k_{z}\right) \mathrm{e}^{-\mathrm{i} k_{z} z} d k_{z}
$$

corresponding to a continuous integral along the transformed variable $k_{z}$. Assuming an infinite number of virtual sources, equally spaced $\mathrm{L}$ along the $\mathrm{z}$ axis, this integral can be approximated as a discrete summation, and written as

$$
P=\frac{2 \pi}{L} \sum_{m=-M}^{M} p\left(k_{z m}\right) \mathrm{e}^{-\mathrm{i} k_{z m} z}, \text { where } k_{z m}=\frac{2 \pi}{L} m .
$$

For this case, the fields generated by a sound source located at point $\mathbf{x}_{0}$ of coordinates $\left(x_{0}, y_{0}\right)$, and for a given axial wavenumber $k_{z}$, can be given, in terms of pressure and particle velocities generated at any receiver located at point $\mathbf{x}$ of coordinates $(x, y)$, as

$$
\begin{gathered}
G\left(\mathbf{x}, \mathbf{x}_{0}, k_{f}\right)=-\frac{\mathrm{i}}{4} \mathrm{H}_{0}^{(2)}\left(k_{f} r\right) \\
H\left(\mathbf{x}, \mathbf{x}_{0}, k_{f}, \vec{n}\right)=\frac{k_{f}}{-4 \rho \omega} \mathrm{H}_{1}^{(2)}\left(k_{f} r\right) \frac{\partial r}{\partial \vec{n}},
\end{gathered}
$$

where $r=\sqrt{\left(x-x_{0}\right)^{2}+\left(y-y_{0}\right)^{2}}$, and $\vec{n}$ represents the direction along which the particle velocity is to be calculated. 


\subsection{MFS formulation}

In the MFS, the solution of the problem is approximated by a linear combination of fundamental solutions. To formulate the method, consider a generic $2.5 \mathrm{D}$ problem governed by equation (2) where the problem's physical boundary $\Gamma$ is subjected to Neumann boundary conditions, as defined by:

$$
-\frac{1}{\mathrm{i} \rho \omega} \frac{\partial}{\partial \vec{n}} p=v_{K} .
$$

To allow obtaining a solution for the problem, consider a set of NS virtual sources located outside the field of analysis, and assume that the pressure field at any domain point $\mathbf{X}$ can be represented by a linear combination of the effects of the NS sources positioned at points $\mathbf{x}_{j}$ so that

$$
p\left(\mathbf{x}, k_{f}\right)=\sum_{j=1}^{N S} Q_{j} G\left(\mathbf{x}, \mathbf{x}_{j}, k_{f}\right),
$$

where $Q_{j}$ is an amplitude factor associated with each of the virtual sources, and which is, a-priori, unknown.

For the problem under study, and given such representation of the pressure field, consider, additionally, a set of NC collocation points distributed along the boundary $\Gamma$. Imposing the desired boundary conditions at each of the NC collocation points, a set of equations can be obtained in the form:

$$
\sum_{j=1}^{N S} Q_{j} H\left(\mathbf{x}_{i}, \mathbf{x}_{j}, k, \vec{n}\right)=v_{K, i} \text { for each } \mathbf{x}_{i} \text { at } \Gamma,
$$

where $v_{K, i}$ are the normal particle velocity values to be prescribed at each collocation point $i$. Establishing these equations, a system with $\mathrm{NC}$ equations on NS unknowns can be written, allowing the calculation of the unknown amplitude factors $Q_{j}$. Evidently, other types of boundary conditions may be imposed along the boundary, although in the scope of the present work only Neumann conditions are considered.

\section{Adaptive-Cross-Approximation}

The Adaptive-Cross-Approximation (ACA) technique is here used to considerably improve the efficiency of the MFS model, rendering it faster and requiring less computer resources (RAM). The ACA is applied to approximate blocks of the MFS matrix, requiring, for that purpose, the calculation of only a much reduced number of rows and columns of that block, thus consisting of a low-rank approximation. The approximated block $\mathrm{A}$ is written in the form:

$$
A \approx S_{k}=U V^{T}
$$


where $U$ and $V$ are obtained from a limited number of rows and columns of $A$.

The calculation of the approximation can be seen as a recursive algorithm, in which a residual matrix $R_{j}$ and an approximation matrix $S_{j}$ at step j are used, assuming that $R_{j}+S_{j}=A$. For the approximation of a $\mathrm{m} \times \mathrm{n}$ matrix:

- At the beginning, the residual matrix is $R_{0}=A$, while the approximation of $A$ is given by $S_{0}=0$;

- For each subsequent step $\mathrm{k}=0,1,2,3, \ldots$,

○ The $i_{k+1}$ row of the original matrix $\mathrm{A}$ is generated $a=A_{i_{k+1}, 1: n}$;

- The row of the residual matrix is calculated as:

- $R_{i_{k+1}, 1: n}^{k}=a-\left(\sum_{l=1}^{k} u_{i_{k+1}, l} v_{1: n, l}\right)^{T}$

○ The pivot column $j_{k+1}$ is identified as the one containing the maximum value of $\left|R_{i_{k+1}, 1: n}^{k}\right|$ and the corresponding column of $A$ is generated $\left(a=A_{1: m, j_{k+1}}\right)$;

○ A normalizing constant is computed as $\gamma_{k+1}=\left(R_{i_{k+1}, j_{k+1}}^{k}\right)^{-1}$;

○ The column of the residual matrix is calculated as:

- $R_{1: m, j_{k+1}}^{k}=a-\sum_{l=1}^{k} v_{j_{k+1}, l} u_{1: m, l}$

- Matrices $U$ and $V$ are updated:

- $u_{1: n, k+1}=\gamma_{k+1}\left(R_{i_{k+1}, 1: n}^{k}\right)^{T}$

- $v_{1: m, k+1}=R_{1: m, j_{k+1}}^{k}$

○ The new pivot row $j_{k+2}$ is determined as the one containing the maximum value of $\left|R_{1: m, j_{k+1}}^{k}\right|$, and the recursive process continues to the first step.

The recursive algorithm is stopped when the variation of the norm of the approximation matrix $S$ is less than a given tolerance. Details on the procedure can be found in Rjasanow [10].

An important advantage of the ACA technique is that it can be applied to different physical problems, being based on the original kernels that originate the matrix to be approximated. Thus, its application can reuse existing codes, which is a significant advantage when compared to other alternatives (such as the Fast Multipole technique, for example).

It is important to understand that only some of the matrix blocks can be approximated using the ACA, while the remaining must be fully computed using the conventional MFS. To determine which blocks can be approximated, hierarchical matrices ( $H$ matrices) are used, built by successive geometrical partition of the physical domain in smaller lower level subdomains (clusters). The subdivision stops when a given cluster contains less than a given number of elements. In the present paper, since the purpose is to analyze sonic crystal 
structures composed of multiple scatterers with similar geometry, the subdivision is performed until each low level cluster contains only one full scatterer (such cluster is named a "leaf"), as illustrated in Figure 1. Following this subdivision, the scatterers must be reordered, so that nodes geometrically far away from each other also have indices with a large offset.

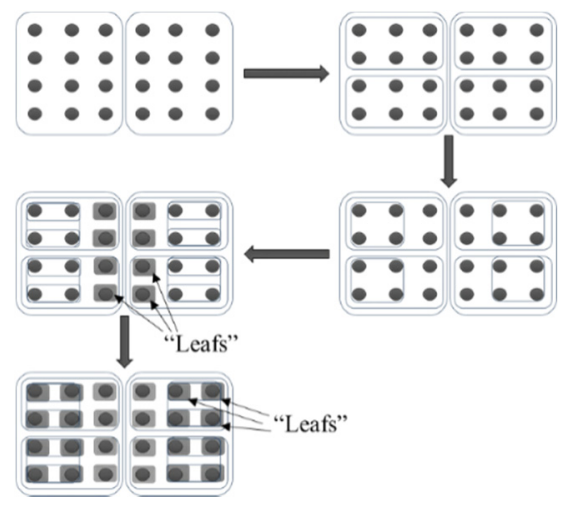

Figure 1: Sequential subdivision of the domain in smaller clusters, until each low-level cluster contains only one scatterer ("leaf").

To check if each matrix block can be approximated using the ACA, at each subdivision step, the distance between blocks is evaluated, and a simple criterion of admissibility is used:

$$
2 \min \left(r_{i}, r_{j}\right)<\eta\left|\mathbf{x}_{i}-\mathbf{x}_{j}\right|
$$

where $r_{i}, r_{j}$ are the "radius" of each cluster and $\left|\mathbf{x}_{i}-\mathbf{x}_{j}\right|$ is the geometrical distance between their centers.

To exploit the full advantages of such approach, it is also necessary to solve the corresponding equation system by means of an iterative solver. In the present work, the GMRES iterative solver has been used, together with a block preconditioner (to accelerate convergence) based on the non-admissible blocks of the matrix.

\section{Verification and performance of the 2.5D ACA-MFS model}

In order to verify the proposed ACA-MFS model and to assess its performance, several cases were analysed. In this section, some illustrative results are presented, concerning a scenario in which a sonic crystal is composed of 315 scatterers with circular cross-section of radius $0.01 \mathrm{~m}$, with their centres equally spaced $0.06 \mathrm{~m}$ between, each of them modelled using 20 collocation points. To simulate the generated pressure field, 20 virtual sources are positioned within each of the scatterers, originating a system with 6,300 collocation points and 6,300 virtual sources. The fluid medium is assumed to be air, with a propagation velocity of 
$340 \mathrm{~m} / \mathrm{s}$ and a density of $1.22 \mathrm{~kg} / \mathrm{m}^{3}$, and is excited by a source positioned at $\mathrm{x}=-0.5 \mathrm{~m}$ and $\mathrm{y}=0.6 \mathrm{~m}$. The acoustic pressure response is computed at two receivers, located at $\mathrm{x}=0.7 \mathrm{~m}$ and $\mathrm{y}=0.75 \mathrm{~m}$ and $\mathrm{at}=0.5 \mathrm{~m}$ and $\mathrm{y}=0.45 \mathrm{~m}$. This system is schematically illustrated in Figure 2.

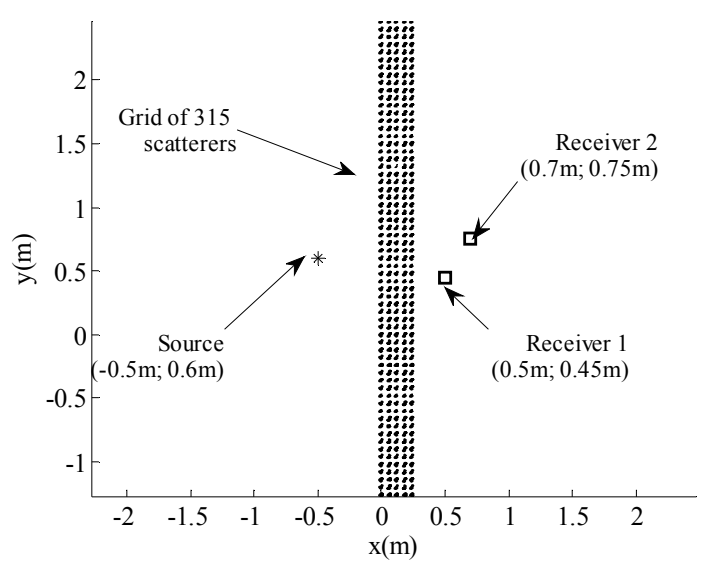

Figure 2: Schematic representation of the acoustic system used for the verification.

As described in section 2, an ACA algorithm is used to assemble the MFS matrix, involving the definition of admissible and non-admissible matrix blocks. Figure 3 represents the block-structure of the system matrix, with darker grey patches representing non-admissible blocks and white patches corresponding to admissible blocks (approximated with ACA). It is clear that the largest part of the matrix can be approximated, and only a small number of diagonal blocks needs to be exactly calculated using the standard MFS.

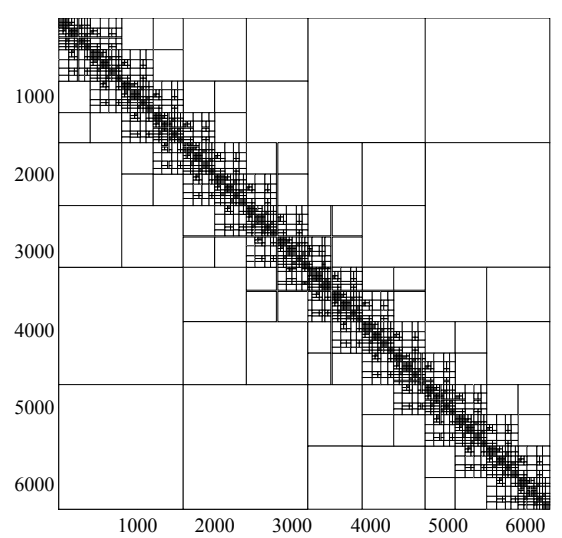

Figure 3: Matrix representation, with darker patches corresponding to nonadmissible blocks. 
To first check the correctness of the results computed using the ACA-MFS, the acoustic pressure is calculated at receivers 1 and 2 (see Figure 2), using both the proposed method and the standard MFS, for a complete range of frequencies from $100 \mathrm{~Hz}$ to $5,000 \mathrm{~Hz}$. Results calculated for two different values of $k_{z}$ are presented in Figure 4, namely for $k_{z}=0.0 \mathrm{rad} / \mathrm{m}$ and for $k_{z}=50.0 \mathrm{rad} / \mathrm{m}$. As can be seen in all presented plots, there is a perfect match between the solutions provided by the standard and by the ACA versions of the MFS at both receivers. It should be mentioned that the ACA-MFS results presented in Figure 4 are calculated considering the use of a pre-conditioner for the GMRES iterative solver, built by just considering the non-admissible blocks of the system matrix. For that purpose, and due to their structure and very limited number of non-zero values in this preconditioner matrix, it is stored as a sparse matrix.
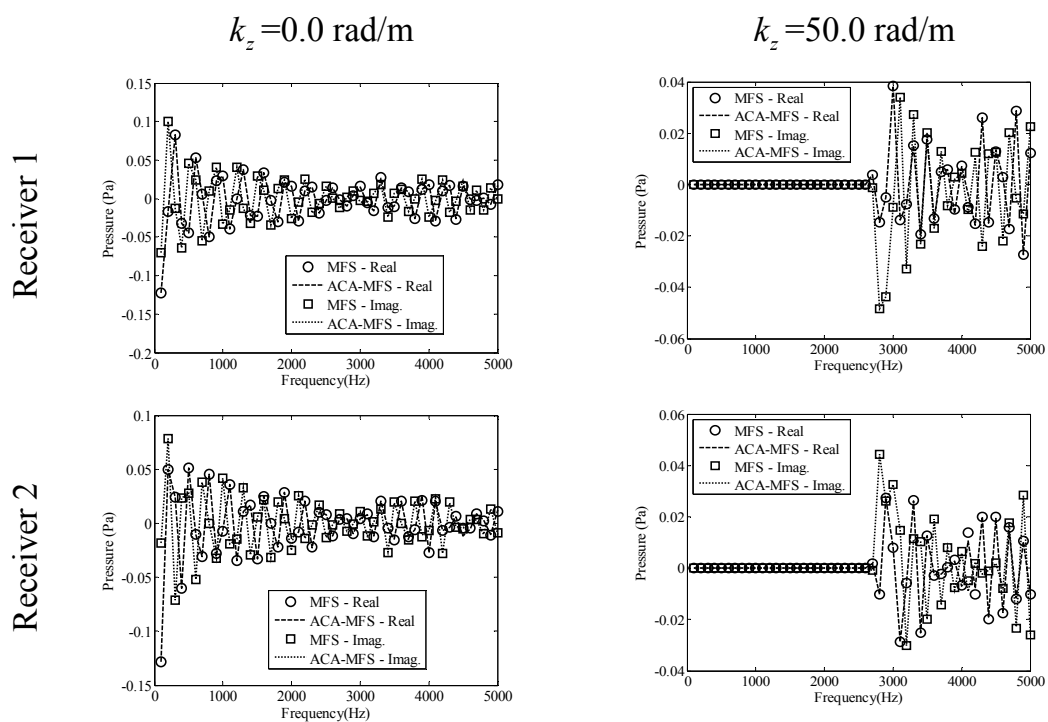

Figure 4: Comparison of the pressure calculated at two receiver points, using the MFS and the proposed ACA-MFS. The left column corresponds to $k_{z}=0.0 \mathrm{rad} / \mathrm{m}$ and the right column to $k_{z}=50.0 \mathrm{rad} / \mathrm{m}$.

An important aspect to be addressed in the present study is related to the computational performance of the proposed ACA-MFS algorithm. To assess the computational performance of the algorithm, several tests were performed, and results in terms of the computational time (in percentage with respect to the time required by the standard MFS) and number of GMRES iterations are illustrated in Figure 5, once again for the two values of $k_{z}$ indicated above. In that figure, results are presented considering different approaches on the use of a pre-conditioner for the GMRES solution. The following cases are considered: the sparse preconditioner described above, built as a sparse matrix containing all non-admissible blocks; a pre-conditioner built as the incomplete LU factorization of the sparse 


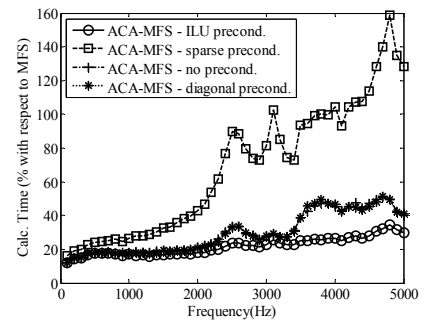

(a1)
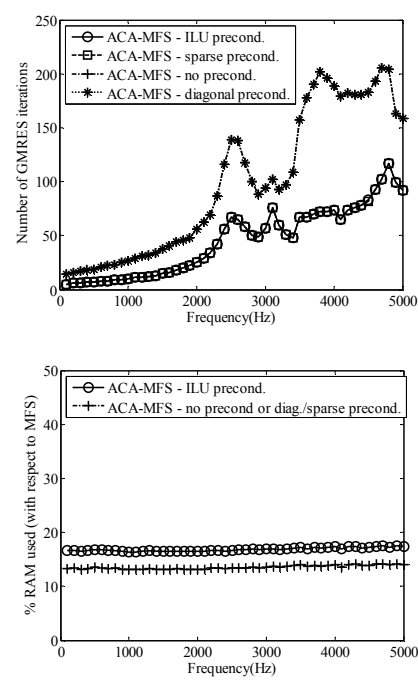

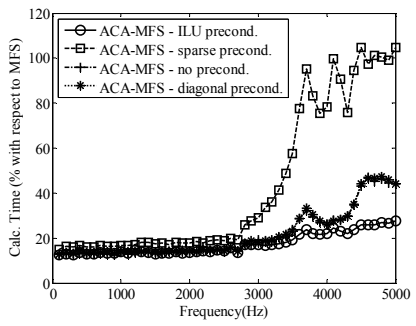

(a2)
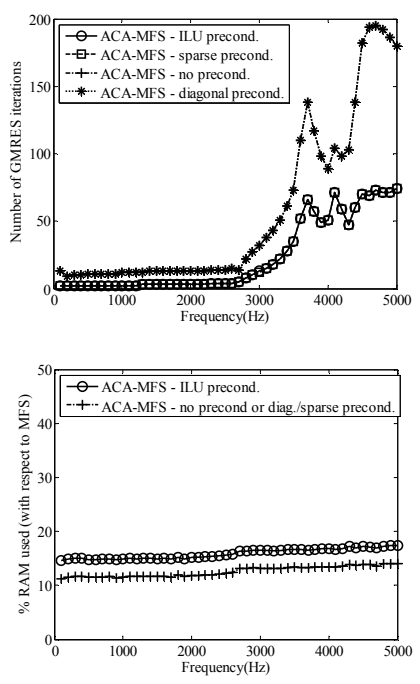

(c2)

Figure 5: Calculation times, number of iterations of the GMRES solver, and RAM memory usage for the ACA-MFS with different preconditioners. Left column ((a1), (b1), (c1)) corresponds to $k_{z}=0.0 \mathrm{rad} / \mathrm{m}$ and right column ((a2), (b2), (c2)) to $k_{z}=50.0 \mathrm{rad} / \mathrm{m}$.

pre-conditioner; a diagonal pre-conditioner built just considering the main diagonal of the system matrix; no pre-conditioner.

Observing the plotted results, it can be seen that all pre-conditioners reach convergence at all frequencies, with a clear tendency for the computational time to increase with the frequency. For the sparse pre-conditioner, however, very large computational times seem to occur for the higher frequencies, and in particular for $k_{z}=0.0 \mathrm{rad} / \mathrm{m}$, even surpassing the original MFS algorithm (reaching a maximum of $160 \%$ of the computational time of the standard MFS). The explanation for this behaviour is related to the additional solution time required for the direct solution of $P y=X$ ( $P$ being the preconditioner matrix). However, if an incomplete LU factorization of $P$ is used instead, the procedure is rendered much more efficient, and the solution time decreases to, at most, $30 \%$ of the time required by the standard MFS. Observing the same plots, it is interesting to note that both the 
sparse pre-conditioner and the ILU pre-conditioner require exactly the same number of iterations at all frequencies, although with very different calculation times. Without using any pre-conditioner, convergence was also reached, although at the cost of a higher number of iterations. The effect of using a diagonal preconditioner seem to be negligible, with the same calculation times and number of iterations as for the case without any pre-conditioner. Very similar conclusions can be drawn from the results of both values of $k_{z}$, although for $k_{z}=50.0 \mathrm{rad} / \mathrm{m}$ seems to require less iterations for convergence at lower frequencies; this may be justified by the fact that an almost null response is obtained for those frequencies and for the higher $k_{z}$, as can be seen in Figure 4.

The last line of Figure 5 presents the amount of RAM required by the different versions of the ACA-MFS algorithm, as a percentage of the RAM required by the standard MFS. The extra memory required when using the ILU pre-conditioner can be justified by the fact that it requires the $\mathrm{L}$ and $\mathrm{U}$ matrices to be stored. However, it can be seen that this additional amount of RAM is not very significant, and the method is still very efficient for that case in what concerns the RAM usage when compared to the standard MFS.

Several additional tests were performed using the proposed ACA-MFS, namely to understand its behaviour for larger problems. Figure 6 illustrates results computed for the original problem, and for problems with 525 and 1,050 scatterers. The three analysed configurations thus include 6,300, 10,500 and 21,000 collocation points, and computations were performed for $k_{z}=10.0 \mathrm{rad} / \mathrm{m}$ and a frequency of $2,500 \mathrm{~Hz}$, using the ILU pre-conditioner. The three plots present the amount of RAM used by the ACA-MFS and by the standard MFS, their calculation times, and the number of GMRES iterations required to reach a solution (only for ACA-MFS). These results indicate that the previously observed computational gains become even more evident and significant when larger problems are modelled, with amounts of RAM and computational times being less than $10 \%$ of those required by the original method. If the number of iterations is analysed, a progressive increase in the number of iterations is observed as the problem size increases.

\section{Conclusions}

In this paper, a strategy based on the use of the MFS was proposed to tackle large problems of sound propagation around 3D sonic crystals with constant geometry along the axis of the scatterers (2.5D). The problem is solved in the frequency domain, and the sound field is synthesized as a summation of simpler 2D problems. To allow the solution of large-scale problems, with a high number of scatterers, an Adaptive-Cross-Approximation approach was proposed and incorporated in the MFS algorithm. Test cases were presented, illustrating the main features of the method, and evidencing its efficiency. Results presented for different values of the wavenumber in the direction in which the cross-section does not vary $\left(k_{z}\right)$ revealed the good accuracy and stability of the method. When 


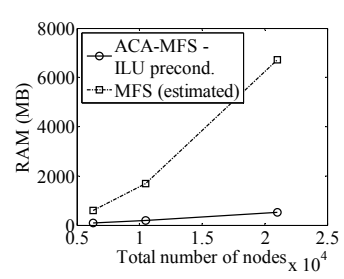

(a)

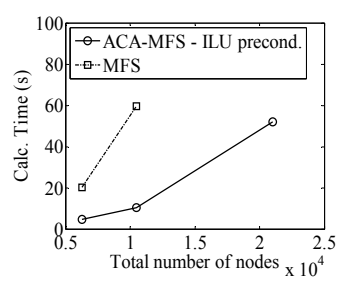

(b)

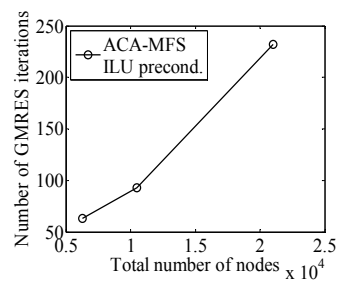

(c)

Figure 6: Results computed for different problem sizes, considering $k_{z}=10.0 \mathrm{rad} / \mathrm{m}$ and a frequency of $2500 \mathrm{~Hz}$ : (a) required RAM;

(b) Calculation time; (c) Number of GMRES iterations.

compared to the standard MFS methodology, a performance improvement of up to ten times was observed, with this improvement becoming more expressive as larger problems were considered. Different pre-conditioner schemes were considered and analysed, with the use of an ILU pre-conditioner revealing good convergence properties. In general, the methodology and the proposed implementation scheme seem to be very adequate for the analysis of sound propagation in the presence of sonic crystal structures.

\section{Acknowledgements}

The financial support from CNPq (Conselho Nacional de Desenvolvimento Científico e Tecnológico) and FAPEMIG (Fundação de Amparo à Pesquisa do Estado de Minas Gerais) is gratefully acknowledged. The support of FCT (Fundação para a Ciência e a Tecnologia) and COMPETE, through research project PTDC/ECM-COM/1438/2012, is also acknowledged.

\section{References}

[1] Wu, L.-Y., Chen, L.-W. \& Liu, C.-M., Acoustic pressure in cavity of variously sized two-dimensional sonic crystal with various filling fraction, Phys. Lett. A, 373, pp. 1189-1195, 2009.

[2] Vasseur, J.O., Deymier, P.A., Djafari-Rouhani, B., Pennec, Y. \& HladkyHennion, A.-C., Absolute forbidden bands and waveguiding in twodimensional phononic crystal plates, Phys. Rev. B, 77, p. 085415, 2008.

[3] Sánchez-Pérez, J.V., Rubio, C., Martínez-Sala, R., Sánchez-Grandia, R. \& Gómez, V., Acoustic barriers based on periodic arrays of scatterers, Appl. Phys. Lett., 81, p. 5240, 2002.

[4] Castiñeira-Ibáñez, S., Rubio, C., Romero-García, V., Sánchez-Pérez, J.V. \& García-Raffi, L.M., Design, Manufacture and Characterization of an Acoustic Barrier Made of Multi-Phenomena Cylindrical Scatterers Arranged in a Fractal-Based Geometry, Archives of Acoustics, 37(4), pp. 455-462, 2012. 
[5] Kafesaki, M. \& Economou, E.N., Multiple-scattering theory for threedimensional periodic acoustic composites, Phys. Rev, B, 60(17), p. 11993, 1999.

[6] Liu, Z., Chan, C.T., Sheng, P., Goertzen, A.L. \& Page, J.H., Elastic wave scattering by periodic structures of spherical objects: Theory and experiment, Phys. Rev. B, 62(4), p. 2446, 2000.

[7] Yang, S., Page, J.H., Liu, Z., Cowan, M.L., Chan, C.T. \& Sheng, P., Focusing of Sound in a 3D Phononic Crystal, Phys. Rev. Letters, 93(2), p. 024301, 2004.

[8] Martins, M., Godinho, L. \& Picado-Santos, L., Numerical Evaluation of Sound Attenuation Provided by Periodic Structures, Archives of Acoustics, 38(4), pp. 503-516, 2013.

[9] Santos, P.G., Carbajo, J., Godinho, L. \& Ramis, J., Sound Propagation Analysis on Sonic Crystal Elastic Structures using the Method of Fundamental Solutions (MFS), CMC: Computers, Materials \& Continua, 43(2), pp. 109-136, 2014.

[10] Rjasanow, S., Adaptive cross approximation of dense matrices, Proc. Int. Association for Boundary Element Methods, pp. 1-12, 2002.

[11] Bebendorf, M., Hierarchical Matrices, A Means to Efficiently Solve Elliptic Boundary Value Problems. Springer Berlin, Heidelberg, 2008. 